\title{
Can urinary indolylacroylglycine levels be used to determine whether children with autism will benefit from dietary intervention?
}

\author{
Julie Wilson ${ }^{1,2}$, Barry Wright ${ }^{3,4}$, Sandra Jost ${ }^{4,5}$, Robert Smith ${ }^{6}$, Helen Pearce ${ }^{7}$ and Sally Richardson ${ }^{8}$
}

BACKGROUND: An increase in urinary indolyl-3-acryloylglycine (IAG) has been reported in children with autism spectrum disorders (ASD) who suffer with bowel problems in comparison to ASD children without gastrointestinal (GI) problems. The case for dietary intervention for ASD children with Gl symptoms might be strengthened were such a difference to be autism-specific.

METHODS: Quantitative analysis of urinary IAG levels was performed for 53 children on the autism spectrum and 146 age-matched controls. The parents of each child were asked to provide information on bowel symptoms experienced by the child and their eating habits over a period of $2 \mathrm{wk}$.

RESULTS: We find no significant difference in urinary IAG levels between the ASD children with Gl problems and ASD children without Gl problems. Although we see some difference between ASD children with Gl problems and controls in mainstream schools with Gl problems, the difference between non-autistic children with other developmental disorders and controls in mainstream schools is more significant so that any difference is not autism-specific. We find a strong correlation between bowel symptoms and diet problems in ASD children, especially idiosyncratic feeding behavior and we show that ASD children suffering from multiple bowel symptoms tend to be those who also have dietary problems.

CONCLUSION: We found no evidence to support the hypothesis that children with ASD who suffer with bowel problems have increased levels of urinary IAG in comparison to children with ASD who do not have gastrointestinal problems.

A utism is a complex disorder characterized by language and communication difficulties, qualitative social reciprocity and restricted, repetitive, and stereotyped patterns of behavior, interests, and activities (1). Despite extensive research based on theories ranging from changes in brain structure (2) to genetic differences and metabolic disturbances (3), the etiology is still unclear as no consistent pathophysiology for all autism spectrum disorders (ASDs) can be established. Several studies have been based on the hypothesis that some symptoms of autism may be associated with an excess of opioid-like peptides derived from dietary sources (4). Such proposals offer the potential for biomedical intervention, but conflicting evidence has been produced related to this "opioid excess theory" with no evidence of the neuropeptides found in children with or without ASD in the targeted analysis of Dettmer and associates (5).

Although Kuddo and Nelson concluded that gastrointestinal problems are much less frequent in children with autism than pediatric gastroenterology clinics report (6), several authors have found a strong association between bowel problems and autism in community samples (7-9). Specific symptoms, such as diarrhea, constipation, foul-smelling stools, flatulence, abdominal bloating, and discomfort have been identified and compared between children with autism and their healthy siblings $(10,11)$. In addition to an increased incidence of constipation, more issues with feeding and food selectivity have been reported for children with autism, suggesting the higher incidence of GI symptoms may be neurobehavioral, although a primary organic gastrointestinal origin cannot be ruled out (12). In their comparison, Chaidez and coworkers found that parents of children with ASD or developmental delay reported more food dislikes (selectivity), as well as more GI symptoms not explained by other factors, than parents of typically developing children (9). Their results also show significantly higher incidence of behavioral problems (irritability, social withdrawal, stereotypy, and hyperactivity) in ASD children with GI problems than those without symptoms. A meta-analysis involving 15 studies also concluded that children with ASD experience significantly more GI symptoms than comparison groups with higher rates of diarrhea, constipation and abdominal pain, but no evidence of a unique pathology in ASD (13). Regardless of cause or effect, the association between diet and autism has been used to suggest treatment in the form of dietary intervention. In particular, some authors hypothesize that gluten- and casein-derived peptides may trigger an immune response resulting in GI symptoms (14). It has

\footnotetext{
'Department of Mathematics, University of York, York, UK; ${ }^{2}$ Department of Chemistry, University of York, York, UK; ${ }^{3} \mathrm{Hull}$ York Medical School, University of York, York, UK; ${ }^{4}$ Leeds and York Partnership NHS Foundation Trust, Leeds, UK; ${ }^{5}$ Department of Health Science, University of York, York, UK; ${ }^{6}$ York Teaching Hospital NHS Foundation Trust, York, UK; ${ }^{7}$ Tees, Esk and Wear Valleys NHS Foundation Trust, Middlesbrough, UK; ${ }^{8}$ University College Hospital, London, UK. Correspondence: Julie Wilson (julie.wilson@york.ac.uk) Received 10 April 2016; accepted 9 October 2016; advance online publication 25 January 2017. doi:10.1038/pr.2016.256
} 
been noted that, for the majority of children diagnosed with autism, symptoms were observed before 18 mo of age, giving a limited time period over which specific foods, particularly gluten, would have been eaten although the milk protein, casein, would have been ingested since birth (15). Although no largescale randomized controlled trials have taken place, some authors suggest that gluten-free and/or casein-free diets can be effective in some children with autism $(16,17)$. However, the findings are not reproduced in other studies $(18,19)$ and a recent double-blind trial found no significant effect on behavior or autism symptoms (20). Black and coworkers found no evidence that, before their diagnosis, children with autism were more likely to have had gastrointestinal disorders than children without autism (21). Although a decrease in urinary peptide secretion has been reported in response to diet as well as improvement in behavior and a decrease in epileptic seizures (22), it has also been observed that the evidence is weak and that gluten-free and/or casein-free diets should only be recommended after intolerance or allergy to foods containing the allergens excluded in these diets has been diagnosed (23). Faddy or selective eating (also known as perseverant eating) is often associated with ASDs and many children with autism reject foods based on their texture, color or other characteristics, potentially leading to nutritional deficiencies (24). A comprehensive meta-analysis, involving 17 studies with a comparison group, revealed that children with ASD experience significantly more feeding problems and have significantly lower intake of calcium and protein (25). This risk may increase if further dietary restrictions are imposed on children with ASD. Until specific evidence-based guidelines have been developed, Buie and coworkers recommend that general pediatric guidelines for diagnostic evaluation of abdominal pain, chronic constipation, and gastroesophageal reflux disease be adapted for children with ASDs (26). Sharp and colleagues encourage clinicians to assess feeding problems in children with ASD and to screen for nutritional deficiencies as well as educating caregivers in the potential consequences of following elimination diets (25).

In the context of proposed bowel and metabolism problems in autism, some have suggested that the metabolite, indolyl3 -acryloylglycine (IAG), has been found in the urine of children with ASD, and may be a putative marker for autism and tryptophan metabolism problems (27). However, this study had no comparison to a control group to establish its specificity to the disorder. In a comparative study, IAG was proposed as a putative diagnostic marker for ASD (28). However, Wright and associates found no statistically significant difference in the levels of urinary IAG between children with ASD and controls without ASD (29). More recently, a link between gastrointestinal (GI) dysfunction in autism and urinary IAG has been suggested, with IAG levels raised only in those ASD children exhibiting bowel symptoms (30). Furthermore, as no such difference was found between control children with and without bowel problems, these authors suggest that increased IAG levels could be autism-specific. If true, such findings could support the hypothesis that problems with digestion lead to symptoms of autism arising from an excess of opioid-like peptides and that dietary intervention might help ASD children with GI symptoms.

Here, we investigate the hypothesis that children with ASD who suffer with bowel problems have increased levels of urinary IAG in comparison to children with ASD who do not have gastrointestinal problems. We also consider the relationship between bowel symptoms, diet and dietary problems and faddy eating in ASD children.

\section{METHODS}

\section{Data Collection}

A leaflet for parents and children was prepared to explain sample collection procedures using standardized equipment. Urine samples were blinded with code numbers and delivered from one of several points of collection to the Department of Chemical Pathology at York Hospital within $24 \mathrm{~h}$ and stored at $-20{ }^{\circ} \mathrm{C}$. Each urine sample was divided into two parts. The first was used to perform quantitative analysis of IAG and the second for determination of the creatinine concentration using a standard method (31) on an Hitachi 917 analyzer (Boehringer, Mannheim, Germany). IAG:creatinine ratios were calculated to control for variations caused by body mass, urine concentration, and other metabolic factors. IAG was synthesized by a method adapted from (32), which involved esterification of indole3 -acrylic acid with glycine methyl ester using dicyclohexylcarbodiimide and hydroxybenzotriazole in dichloromethane. In addition, a dideuterated analogue of IAG was synthesized by the same route according to a method by Tilley and associates (33). Analysis of urine samples for IAG was effected by using high performance liquid chromatography with tandem mass spectrometric detection. Dideuterated IAG was used as an internal standard. The method was validated according to internationally recognized standards (34) to ensure precision, accuracy, and specificity.

As the distribution of IAG levels (measured in mmol) was skewed to the left, a constant of 0.001 was added before taking the natural logarithm to normalize the distribution. An IAG: creatinine ratio was calculated to allow for variations in urine concentration and the ratios also transformed by adding a constant of 0.001 and taking the natural logarithm. After the transformation, Shapiro-Wilk tests showed that the data for each group were plausibly normal, making the use of Student's $t$-tests valid. In addition we report results for the nonparametric Wilcoxon rank sum test, which does not require the data to be normally distributed.

The age distribution for the ASD group and for the mainstream school control group is very similar and neither group shows correlation between age and IAG levels (Table 1). Therefore, although

Table 1. Summary of IAG level comparisons

\begin{tabular}{lcc}
\hline & Student's t-test & Wilcoxon test \\
\hline ASD-GI vs. ASD-NGI & 0.199 & 0.131 \\
MAIN-GI vs. MAIN-NGI & 0.084 & 0.100 \\
SPEC-GI vs. SPEC-NGI & 0.367 & 0.387 \\
CON-GI vs. CON-NGI & 0.927 & 0.986 \\
ASD-GI vs. MAIN-GI & $0.035^{\mathrm{a}}$ & 0.117 \\
SPEC-GI vs. MAIN-GI & $0.004^{\mathrm{a}}$ & $0.002^{\mathrm{a}}$ \\
ASD-GI vs. SPEC-GI & 0.457 & 0.246 \\
ASD-GI vs. CON-GI & 0.596 & 0.742 \\
\hline
\end{tabular}

ASD-GI, N=29; ASD-NGI, N=22; MAIN-GI, N=14; MAIN-NGI, N=99; CON-GI, N= 26; CON-NGI, N=119; SPEC-GI, N=12; SPEC-NGI, N=20. The children in the special school group suffered from other developmental disorders, but were not diagnosed with autism. aResult significant at the $95 \%$ confidence level.

ASD, autism spectrum disorders; GI, gastrointestinal problems; NGI, no gastrointestinal problems; IAG, indolyl-3-acryloylglycine. 
Wang and colleagues report results for age-adjusted values (30), we have chosen not to perform this data manipulation. However, there are more older children and young adults in the special school control group with a slightly greater negative correlation with age.

A questionnaire on bowel symptoms experienced by the child over a period of $2 \mathrm{wk}$ was given to parents at the time the child's urine sample was given. Standard definitions of symptoms were reviewed including WHO definitions of bowel problems such as diarrhea. A meeting with a group of five consultant general pediatricians and two registrars further defined and agreed symptoms to be included. The following questions on bowel symptoms experienced by the child and noticed by the parent were included in the questionnaire:

A1. Do you have any concerns about your child's bowels?

A2. Does he/she have constipation?

A3. Does he/she have diarrhea?

A4. Does he/she have persistent diarrhea (more than $3 \mathrm{wk}$ and more than three times a day)?

A5. Does he/she complain of recurrent abdominal pain?

A6. Does he/she have problems with recurrent vomiting?

A7. Does he/she complain of abdominal distension or tummy bloating?

A8. Does he/she have excessive flatulence?

A9. Has there ever been any blood present in his her stools?

A10. Have you ever seen a doctor specifically because of a concern that your child may have a bowel disorder?

A11. Have you ever seen a doctor who specializes in bowel disorders?

A12. Has your child been specifically diagnosed as having a bowel disorder?

A13. Have you given your child a treatment because of a bowel problem?

The "bowel score" was calculated as the number of positive responses to these questions.

Questions A2, A3, A5, A7, and A8 correspond to the questions used in the Wang study (30).

Parents were also asked to fill in a questionnaire to provide information on their child's eating habits and preferences during the period over which the bowel data were collected. Parents were asked to assign a number between 0 (not at all) and 6 (definitely) to describe the truth of the following statements:

B1. My child is very particular about what they eat.

B2. My child's preferences lead to a very restricted diet.

B3. My child intensely refuses foodstuffs that they prefer not to eat.

B4. There are factors that affect my child's eating, making food preparation and feeding difficult.

B5. My child shows an obsessive insistence to only eat foods that fit certain criteria.

B6. Foodstuffs have to be strictly separated on the plate for my child to accept and consume them.

B7. My child's appetite varies greatly.

B8. My child closely examines food served to them before accepting and consuming it.

B9. My child eats foodstuffs in one place that they will not eat in another place, despite the food being similar.

B10. My child is not happy eating at restaurants (not fast-food outlets).

B11. My child shows an excessive desire to use condiments (e.g., Ketchup, salt, etc.)

For each child with a full bowel and diet history, a "diet score" was obtained by summing the parental scores for these statements.

In this study, a child is considered to have diet problems if the answer to either of the following questions from the questionnaire is positive:

C1. Are you concerned about the range of foods that your child has in the diet?

C2. Have you ever seen a dietician for dietary advice for your child?

The "diet problem indicator" is therefore zero, if the responses to questions $\mathrm{C} 1$ and $\mathrm{C} 2$ are both negative, and one otherwise.
To determine the effect of various factors related to food types or products on a child's food selectivity, parents were asked to give a score between 0 and 6 to rate the effect of the following factors:

D1. Appearance.

D2. Color

D3. Gimmicks.

D4. Logos.

D5. Packaging.

D6. Smell.

D7. Taste.

D8. Texture.

The "faddiness score" was calculated by summing the parental scores for these statements.

\section{Participants}

Children and young people were recruited for a study into urine metabolites (29) by sending letters of invitation to all cases on the Autism Spectrum Disorders Forum register at York with a diagnosis of childhood autism, atypical autism or Asperger syndrome made using criteria from the World Health Organization International Classification of Diseases system version 10 (35). The diagnosis made through the multidisciplinary multi-agency ASD Forum involved consultant pediatricians, consultant child psychiatrists, consultant child psychologists, educational psychologists and speech and language therapists (36). In cases where the ICD-10 diagnosis could not be independently validated by at least two members of the Forum $(72 \%$ of cases), the diagnosis was supported using the Autism Diagnostic Inventory-Revised (ADI-R) and the Autism Diagnostic Observation Schedule-Generic (ADOS) (37). This is the routine assessment and diagnosis protocol for the local multidisciplinary ASDs Forum.

As controls, for each child or young person with ASD, at least one age and sex matched child or young person without autism was recruited from both a special school and a mainstream school. The children in the special schools group suffered from learning disabilities, but none were diagnosed with or were suspected by parents or teachers as having an autism spectrum disorder. Following consent from head-teachers and governors, the parents were circulated with a standard information leaflet inviting them to participate in the study. Informed consent was obtained from parents. Exclusion criteria included any known metabolic disorder and, for the non-autistic group, any previous assessment for an autism spectrum disorder.

A total of 54 children on the autism spectrum participated, of which 51 had a complete bowel data set (94\%) with 29 suffering from GI problems and 22 without GI problems. Using the estimate of $d=0.97$ from Wright and colleagues for a difference in IAG levels between ASD children and controls (29), we would need 18 children in each group to see a difference of this size at a power of $80 \%$ and significance level of 5\% (two-tailed). Of the 155 controls (comprising 121 in main stream schools, 56 of whom were age, sex and school matched for the autism spectrum children, and 34 in special school), 145 (113 mainstream and 32 special school) had a complete bowel data set $(94 \%)$. Full diet and bowel data were available for 43 children on the autism spectrum (80\%) and 22 control children in special school (65\%). Table 2 shows the gender and age distribution of the participants and Table 3 gives the number of various bowel symptoms reported for the different groups.

Ethics review committee: York Research Ethics Review Committee. REC Ref 04/01/007.

\section{RESULTS}

We compared IAG levels and the results of a bowel symptom questionnaire between a group of children with autism, a control group of normal healthy children and a third group, consisting of children with other developmental disorders attending special schools, but who had not been diagnosed with autism. For each child with a full bowel data set, a positive response to any of questions A2, A3, A5, A7, and A8 on the questionnaire (see Methods) was considered to suffer from 
Table 2. Gender and age distribution of the participants included in the analysis of IAG levels

\begin{tabular}{lccc}
\hline & $\begin{array}{l}\text { ASD } \\
\text { group }\end{array}$ & $\begin{array}{l}\text { Mainstream school } \\
\text { control group }\end{array}$ & $\begin{array}{l}\text { Special school } \\
\text { control group }\end{array}$ \\
\hline Gender & 42 & 65 & 18 \\
Male & 11 & 54 & 16 \\
Female & 1 & 2 & 0 \\
Not given & & & \\
age (years) & 4 & 4 & 0 \\
$0-4$ & 17 & 52 & 4 \\
$5-8$ & 25 & 59 & 10 \\
$9-12$ & 5 & 4 & 15 \\
$13-16$ & 3 & 2 & 5 \\
17-20 & -0.01 & -0.13 & -0.29 \\
Age-IAG correlation & a & & \\
\hline
\end{tabular}

aPearson correlation coefficient between age and $\ln (I \mathrm{AG}+0.001)$ values.

ASD, autism spectrum disorders; IAG, indolyl-3-acryloylglycine.

Table 3. The number of participants providing bowel and diet data with number of various symptoms reported for each group

\begin{tabular}{lccc}
\hline & ASD group & $\begin{array}{c}\text { Mainstream school } \\
\text { control group }\end{array}$ & $\begin{array}{c}\text { Special school } \\
\text { control group }\end{array}$ \\
\hline Bowel data & 51 & 113 & 32 \\
Diet data & 43 & 33 & 22 \\
Gl & 29 & 14 & 12 \\
NGl & 22 & 99 & 20 \\
Gastrointestinal symptoms & & \\
diarrhea & 14 & 6 & 3 \\
constipation & 13 & 5 & 10 \\
abdominal pain & 8 & 8 & 2 \\
bloating & 7 & 4 & 2 \\
flatulence & 12 & 2 & 4 \\
blood in stool & 4 & 1 & 0 \\
\hline
\end{tabular}

ASD, autism spectrum disorders; Gl, gastrointestinal problems; NGI, no gastrointestinal problems.

gastrointestinal problems. These questions correspond to the question used in the study by (30) to determine whether a child had GI problems: "Does your child have any chronic/ongoing gastrointestinal issues i.e. bloating, diarrhea, constipation, excessive flatulence, abdominal pain?" (The comparisons made between groups are summarized in Table 1).

\section{Do ASD Children With GI Symptoms Have Higher Levels of Urinary IAG Than ASD Children Without GI Symptoms?}

We found no significant difference in mean IAG level between the ASD children with GI problems and ASD children without GI problems $(t$-test $P=0.20)$. Similarly, we found no significant difference between the controls with GI problems and controls without GI problems ( $t$-test $P=0.93$ ), (the boxplots in Figure 1 show the distribution of $\ln$ (IAG:creatinine) for the children with ASD and the controls, with and without GI problems in each case). Here, the controls include both mainstream

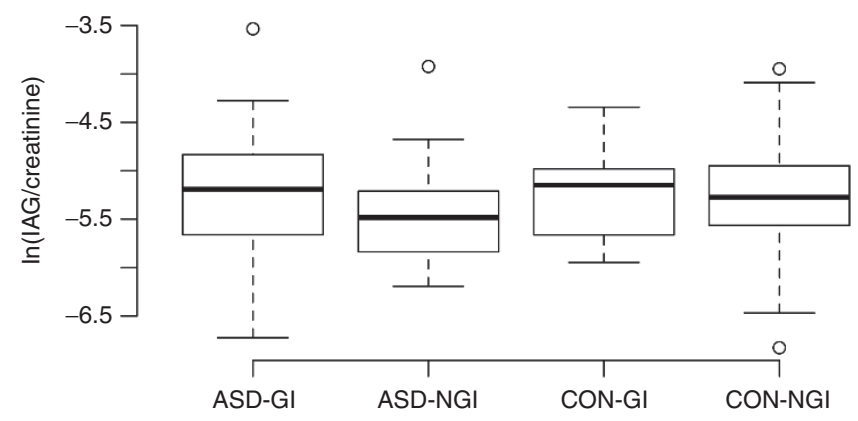

Figure 1. Distribution for the natural logarithm of IAG:creatinine ratio for children with ASD and controls (CON), with and without Gl problems (GI and NGI respectively). In each case the box is determined by the first and third quartiles of the data, so that $50 \%$ of the data lie within the box (the interquartile range), with the thick line showing the median. The dashed lines extend to the lowest and highest values that are within 1.5 times of the interquartile range and data points outside this range are shown individually as circles. ASD, autism spectrum disorders; Gl, gastrointestinal problems; $\mathrm{NGI}$, no gastrointestinal problems; IAG, indolyl-3-acryloylglycine.

and special school groups, but we also found no significant difference between children with and without GI problems in either the mainstream school group $(t$-test $P=0.08)$ or the special school group ( $t$-test $P=0.37$ ) when considered separately.

Stepwise regression with our data starting with the five covariates corresponding to the Wang study leads to a model that only includes constipation and abdominal pain as covariates related to age-adjusted IAG levels. Although the $P$-value of 0.05 might suggest that the regression is just about significant, the $R^{2}$ value of 0.03 shows that the model is a very poor fit, accounting for little of the variance in the data. The low $P$-value is mainly due to a number of unusually high IAG levels having undue influence. We also analyzed the additional bowel data on recurrent vomiting, blood in stools, parental concerns about bowel habit and whether children had ever been assessed or treated by a bowel specialist or diagnosed with a bowel disorder. Again we found no evidence of increased levels of IAG in children with ASD suffering from any particular symptom or subset of symptoms.

\section{Do ASD Children With GI Symptoms Have Higher levels of urinary IAG than control children with Gl symptoms?}

Considering only children with GI problems, a student's $t$-test shows the difference in means between the children with ASD and controls in mainstream schools to be statistically significant $(P=0.04)$ with higher levels in mainstream children (the box-plots in Figure 2 show the distributions of IAG levels for children with gastrointestinal problems). However, a Wilcoxon rank sum test does not give a significant result $(P=$ 0.12 ). Furthermore, the mean for the control children in special schools is also significantly lower than that for controls in mainstream schools ( $t$-test $P=0.004)$. In fact, a $t$-test shows no statistically significant difference between the children with ASD suffering from bowel problems and the control children in special schools suffering from bowel problems $(P=0.46)$ and when the two control groups are combined, there is no statistically significant difference between this group and the children with ASD $(P=0.60)$. 


\section{Articles | Wilson et al.}

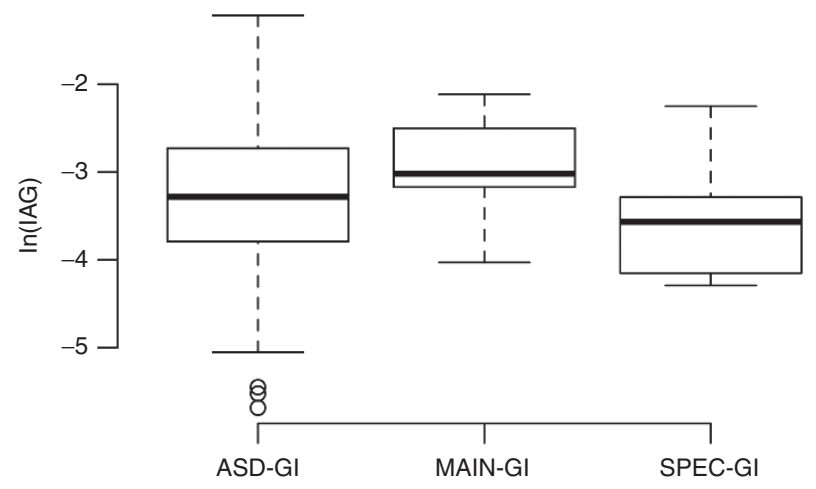

Figure 2. Distribution indolyl-3-acryloylglycine (IAG) levels for children with GI problems. A constant 0.001 was added to IAG levels (measured in $\mathrm{mmol}$ ) before taking the natural logarithm. The boxplots show the distribution for children with ASD (ASD-GI) and controls, in both mainstream (MAIN-GI) and special (SPEC-GI) schools. Although the levels are significantly lower for ASD children than for mainstream school controls ( $t$-test $P=0.035$ ), the levels for children in special schools (without ASD) are also significantly lower than mainstream school controls ( $t$-test $P=0.004)$. ASD, autism spectrum disorders.

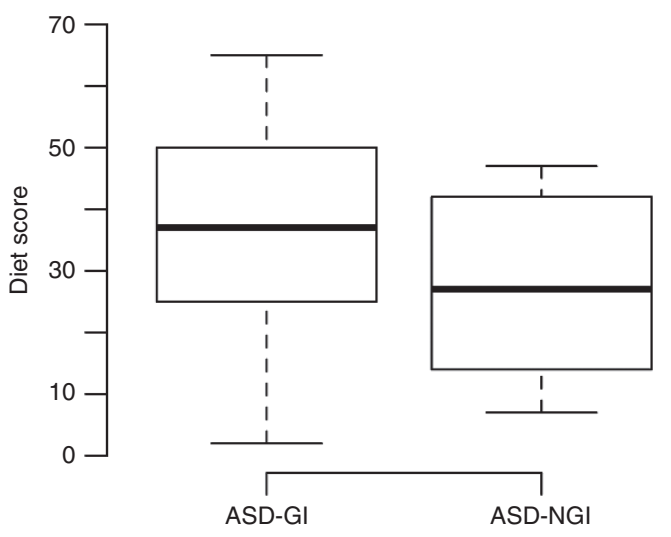

Figure 3. Distribution of diet scores for ASD children with Gl problems (ASD-Gl, $N=25$ ) and ASD children without Gl problems (ASD-NGl, $N=18$ ). ASD, autism spectrum disorders; Gl, gastrointestinal problems; $\mathrm{NGl}$, no gastrointestinal problems.

\section{Do ASD Children With GI Symptoms Have More Diet Issues Than ASD Children Without GI Symptoms?}

For each child with a full bowel and diet history, a diet score was obtained from the level of agreement with statements B1 to $\mathrm{B} 11$ on the dietary questionnaire (Figure 3 shows the distribution of diet scores for children with ASD with GI problems $(n=25)$ and children with ASD without GI problems $(n=18)$. A $t$-test shows the difference between the diet scores for ASD children with and without bowel problems to be statistically significant at the $95 \%$ confidence level $(P=0.01)$. We found no such difference in the control group, but with just 8 of the 22 special school controls with full diet and bowel history having GI problems, the numbers are too small to draw any conclusions.

\section{Do ASD Children With Diet Problems Have More Bowel Problems Than ASD Children Without Diet Problems?}

A child was considered to have dietary problems if the parent was concerned about the range of foods eaten or had seen a

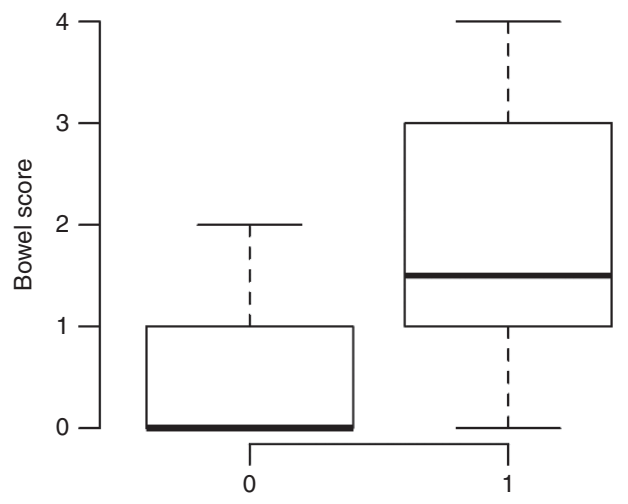

Figure 4. Distribution of bowel scores for children with autism spectrum disorders with diet problems (labeled 1 ) and without diet problems (labeled 0).

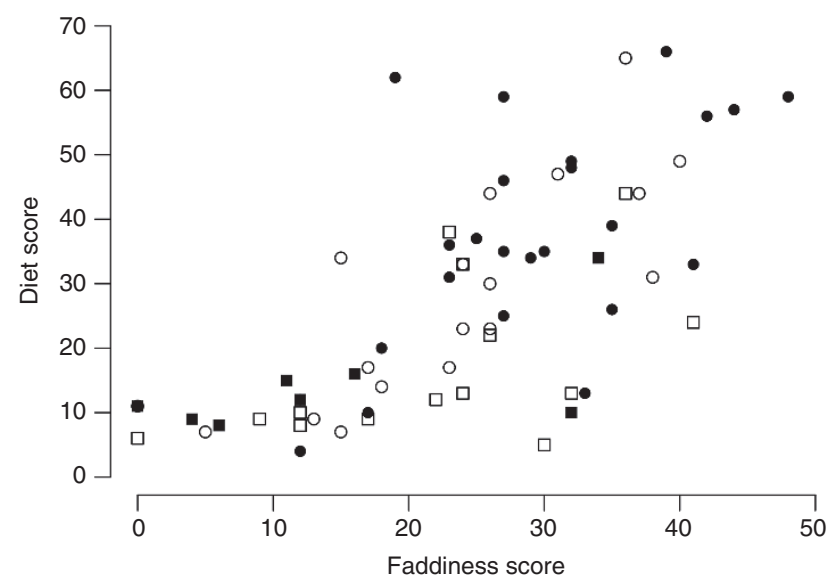

Figure 5. Scores for faddiness plotted against diet scores. Circles denote autism spectrum disorders children and squares denote special school controls with solid symbols representing children suffering gastrointestinal (GI) problems and open symbols representing those without $\mathrm{Gl}$ problems in each case.

dietician for dietary advice for their child. A $t$-test shows a significant difference $(P=0.002)$ in bowel scores for children with ASD with and without dietary problems (the distribution of bowel scores, i.e., the number of reported bowel symptoms, is shown in Figure 4 for ASD children with and without dietary problems). It can be seen that those children reported to be suffering from multiple bowel symptoms tend to be the children with more dietary issues.

\section{Do ASD Children Really Have Faddy Eating Habits?}

Parents were also asked to rate the effect of certain factors related to food types or products (D1 to D8 in the Appendix) on their child's food selectivity with a score between 0 and 6 for each. A "food faddiness score" was calculated by summing these parental scores (Figure 5 shows the faddiness scores plotted against diet scores for each group). There is a strong correlation between faddiness and diet score, particularly for ASD children with GI problems $(r=0.80)$. For ASD children without GI problems, the correlation is somewhat weaker $(r=$ $0.60)$ as it is for children without autism in special schools with 


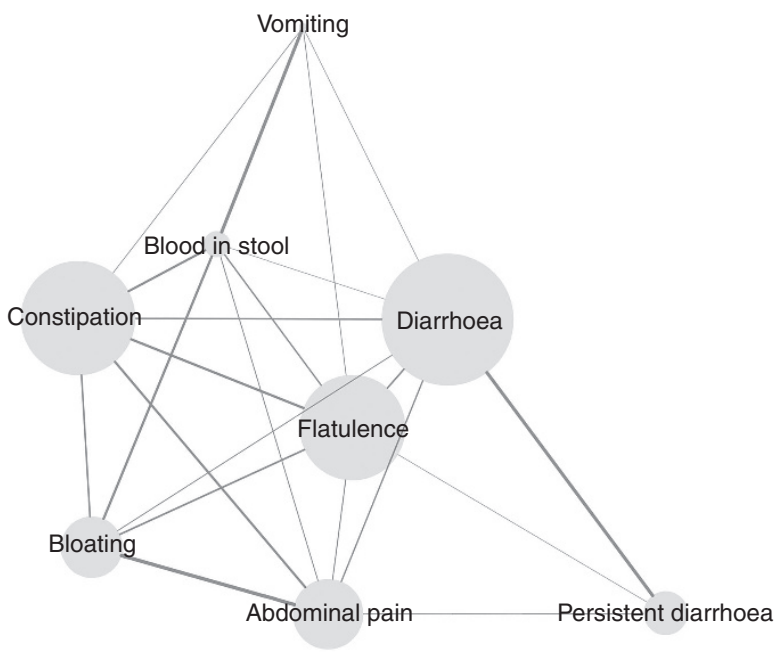

Figure 6. Network analysis on the bowel symptoms for children with autism spectrum disorders. The size of the node is related to the number of occurrences of the various symptoms and the thickness of the lines indicate the strength of the association between symptoms.

or without GI problems ( $r=0.54$ and $r=0.64$ respectively). A $\chi^{2}$ test of the binary diet problem indicator (reflecting a positive response by the parent to either question $\mathrm{C} 1$ or $\mathrm{C} 2$ on the questionnaire) with the binary bowel score (indicating the presence or absence of bowel symptoms) shows a significant association between dietary problems and bowel problems in children with ASD $(P=0.0056)$.

\section{DISCUSSION}

Significant differences in mean IAG:creatinine ratios were proposed in ASD children with on-going GI problems compared with ASD children without GI problems (30). Furthermore, as no significant difference was found between ASD children with GI problems and control children with on-going GI problems, Wang and colleagues concluded that raised IAG levels could be specific to autism. Such a difference could provide support for dietary intervention in those ASD children who also suffer from gastrointestinal problems. However, we found no evidence to support this. There were no statistically significant differences in IAG:creatinine ratios whether only the five questions corresponding to the Wang study were used to determine the presence of GI problems or the additional questions from our more comprehensive bowel questionnaire were included.

If the level of urinary IAG was a useful biomarker for autism, even if only in children experiencing gastrointestinal problems, then a significant difference in urinary IAG between children with GI problems, with and without ASD would be required. That is, IAG levels for children with ASD and GI problems should be compared with children without ASD who have GI problems. Considering only children with GI problems, we find that both the children with ASD and the control children in special schools have lower IAG levels than the controls in mainstream schools, in direct contrast to the reported higher levels in ASD children (30). Moreover, we find no significant difference between IAG levels for ASD children and children with other learning disabilities, confirming the lack of evidence for an autism specific rise in IAG. This reflects the previous finding of Smith and colleagues that both groups show a similar increase in reported bowel symptoms in comparison to a control group in mainstream schools (8). Although bowel symptoms will occur with a wide range in severity, we use a binary indicator as our results rely on parents' perceptions. However, Gorrindo and colleagues investigated parental reports of GI disorders relative to evaluations by pediatric gastroenterologists and found the parents' views to be highly concordant with physicians' diagnosis (38). The finding that children with learning disabilities and GI problems have lower IAG levels than those with GI problems in mainstream schools in mainstream schools is difficult to explain. It is possible that this is to do with dietary intake (with many, but not all children with ASD, also in special schools) or to do with levels of activity leading to different metabolism or to some other common environmental factor. It is noticeable that the participants in the special schools control group tend to be older than those in the other two groups: $46 \%$ of mainstream controls (and 38\% of ASD children), are under $9 \mathrm{y}$ of age in comparison to $29 \%$ of special school controls, whereas only $5 \%$ of mainstream controls (and 15\% of ASD children) are over $12 \mathrm{y}$ of age in comparison to $59 \%$ of special school controls. Although we obtained similar results after adjusting for age using the pooled population as the standard population, it could be that this has some effect.

It is possible that the conflicting results could be due to sibling influence. It is interesting to note that, in the study of Wang and colleagues, $26 \%$ of sibling controls were reported to suffer from GI symptoms, in comparison to just $5 \%$ of their unrelated community controls, whereas $12 \%$ of our mainstream school controls and 38\% of our special school controls report GI symptoms. In our study, 29/51 (57\%) of children with ASD have GI problems compared with 26/57 (46\%) in the Wang study. It is also possible that there are differences due to cohorts suffering from different symptoms, as for example, some children may suffer more from diarrhea and others more from constipation. Molloy and Manning-Courtney found diarrhea to be the most common symptom in ASD children (39), whereas Ibrahim and colleagues report increased incidence of constipation (12). Analysis of our data shows roughly equal numbers of children with ASD suffer from diarrhea $(14 / 51)$ and constipation (13/51) with four children reporting symptoms of both. We performed network analysis using an adjacency matrix obtained from the number of ASD children suffering from pairs of bowel symptoms (Figure 6 shows the strength of the association between different bowel symptoms for children with ASD). It can be seen that excess flatulence is commonly associated with both diarrhea and constipation, that the presence of blood in stools is most often associated with constipation and that abdominal pain is frequently associated with bloating. Instances of persistent diarrhea (more than $3 \mathrm{wk}$ and more than three times a day) and recurrent vomiting were much less frequent. When any reported treatment 
for bowel symptoms was included in the network analysis, this was shown to be most associated with constipation, followed by abdominal pain and bloating, with diarrhea related to treatment to a lesser extent.

We find a strong association between dietary problems and gastrointestinal problems in children with ASD. The direction of this association is not possible to determine with accuracy but there is a considerable body of literature showing that extreme faddiness $(40)$ and poor dietary intake $(41,42)$ in children has a significant impact on bowel habit. It is of course also possible that a primary bowel problem may influence dietary intake (17). However, in many children with ASD no formal bowel pathology has been consistently diagnosed (6). Indeed, Gorrindo and colleagues found bowel problems were not associated with 7 -d food intake but were associated with social and language impairments (38).

\section{Conclusions}

We found no evidence to support the hypothesis that children with ASD who suffer with bowel problems have increased levels of urinary IAG in comparison to children with ASD who do not have gastrointestinal problems. We also found no statistically significant difference in the IAG levels or IAG:creatinine ratios in controls with bowel problems compared to those without such symptoms, whether or not children in mainstream and special schools were considered separately. We find lower levels of urinary IAG in children with ASD suffering from GI problems than in control children in mainstream schools with GI problems, in direct contrast to the findings of Wang and colleagues (30), who report significantly higher levels of IAG in ASD children. Furthermore, we also found significantly lower levels of IAG in the control children in special schools with GI problems in comparison to the control children in mainstream schools with GI problems, so that any difference is not autism specific.

Our research supports the view that GI abnormalities and unusual dietary preferences and behaviors may be associated in some children with autism but it is more likely that this is related to dietary intake, and faddiness and selectivity with secondary nutritional deficits than a primary bowel problem. With this controversy in mind, GI abnormalities should not be considered as a defining characteristic of autism (43).

According to surveys, up to $40 \%$ of children with autism have been placed on special diets at some time (44). These can be dangerous in restricting healthy nutritional intake essential for brain and body development (45). Although some special diets, such as low-sugar diets, can be healthy, casein-free diets, for example, can be a concern due to decreased calcium intake. We urge caution until more definite research can be done into the relation between diet, bowel habit in ASD children and the role of nutritional intake. Although a number of potential biomarkers for autism have been suggested, there is currently insufficient evidence for urinary IAG to provide further insight into the etiology or diagnosis of autism and that there is not sufficient evidence for IAG to be used as a test to recommend dietary intervention whether this is in ASD children in general or in children with autism and GI problems.

\section{ACKNOWLEDGMENTS}

We would like to thank the families who took part in this study. Thanks also to Joel Town who, as an undergraduate project student, co-designed the dietary questionnaire with B.W.

\section{STATEMENT OF FINANCIAL SUPPORT}

No financial assistance was received to support this study.

Disclosure: There are no disclosures to be made regarding financial ties to products in the study or potential/perceived conflicts of interest.

\section{REFERENCES}

1. Baird G, Simonoff E, Pickles A, et al. Prevalence of disorders of the autism spectrum in a population cohort of children in South Thames: the Special Needs and Autism Project (SNAP). Lancet 2006;368:210-5.

2. Stanfield AC, McIntosh AM, Spencer MD, Philip R, Gaur S, Lawrie SM. Towards a neuroanatomy of autism: a systematic review and meta-analysis of structural magnetic resonance imaging studies. Eur Psychiatry 2008;23:289-99.

3. Parellada M, Penzol MJ, Pina L, et al. The neurobiology of autism spectrum disorders. Eur Psychiatry 2014;29:11-9.

4. Elder JH. The gluten-free, casein-free diet in autism: an overview with clinical implications. Nutr Clin Pract 2008;23:583-8.

5. Dettmer K, Hanna D, Whetstone P, Hansen R, Hammock BD. Autism and urinary exogenous neuropeptides: development of an on-line SPE-HPLCtandem mass spectrometry method to test the opioid excess theory. Anal Bioanal Chem 2007;388:1643-51.

6. Kuddo T, Nelson KB. How common are gastrointestinal disorders in children with autism? Curr Opin Pediatr 2003;15:339-43.

7. Valicenti-McDermott M, McVicar K, Rapin I, Wershil BK, Cohen H, Shinnar S. Frequency of gastrointestinal symptoms in children with autistic spectrum disorders and association with family history of autoimmune disease. J Dev Behav Pediatr 2006;27(2 Suppl):S128-36.

8. Smith RA, Farnworth H, Wright B, Allgar V. Are there more bowel symptoms in children with autism compared to normal children and children with other developmental and neurological disorders?: A case control study. Autism 2009;13:343-55.

9. Chaidez V, Hansen RL, Hertz-Picciotto I. Gastrointestinal problems in children with autism, developmental delays or typical development. J Autism Dev Disord 2014;44:1117-27.

10. Horvath K, Papadimitriou JC, Rabsztyn A, Drachenberg C, Tildon JT. Gastrointestinal abnormalities in children with autistic disorder. J Pediatr 1999;135:559-63.

11. Horvath K, Perman JA. Autism and gastrointestinal symptoms. Curr Gastroenterol Rep 2002;4:251-8.

12. Ibrahim SH, Voigt RG, Katusic SK, Weaver AL, Barbaresi WJ. Incidence of gastrointestinal symptoms in children with autism: a population-based study. Pediatrics 2009;124:680-6.

13. McElhanon BO, McCracken C, Karpen S, Sharp WG. Gastrointestinal symptoms in autism spectrum disorder: a meta-analysis. Pediatrics 2014;133:872-83.

14. Pennesi CM, Klein LC. Effectiveness of the gluten-free, casein-free diet for children diagnosed with autism spectrum disorder: based on parental report. Nutr Neurosci 2012;15:85-91.

15. Pavone L, Fiumara A, Bottaro G, Mazzone D, Coleman M. Autism and celiac disease: failure to validate the hypothesis that a link might exist. Biol Psychiatry 1997;42:72-5.

16. Whiteley P, Haracopos D, Knivsberg AM, et al. The ScanBrit randomised, controlled, single-blind study of a gluten- and casein-free dietary intervention for children with autism spectrum disorders. Nutr Neurosci 2010;13:87-100.

17. Jyonouchi H, Sun S, Itokazu N. Innate immunity associated with inflammatory responses and cytokine production against common dietary proteins in patients with autism spectrum disorder. Neuropsychobiology 2002;46:76-84.

18. Robertson MA, Sigalet DL, Holst JJ, Meddings JB, Wood J, Sharkey KA. Intestinal permeability and glucagon-like peptide- 2 in children with autism: a controlled pilot study. J Autism Dev Disord 2008;38:1066-71. 
19. Kemperman RF, Muskiet FD, Boutier AI, Kema IP, Muskiet FA. Brief report: normal intestinal permeability at elevated platelet serotonin levels in a subgroup of children with pervasive developmental disorders in Curaçao (The Netherlands antilles). J Autism Dev Disord 2008;38: 401-6.

20. Hyman SL, Stewart PA, Foley J, et al. The Gluten-Free/Casein-Free Diet: A Double-Blind Challenge Trial in Children with Autism. J Autism Dev Disord 2016;46:205-20.

21. Black C, Kaye JA, Jick H. Relation of childhood gastrointestinal disorders to autism: nested case-control study using data from the UK General Practice Research Database. BMJ 2002;325:419-21.

22. Reichelt KL, Ekrem J, Scott H. Gluten, milk proteins and autism: dietary intervention effects on behavior and peptide secretion. J Appl Nut 1990;42:1-11.

23. Marí-Bauset S, Zazpe I, Mari-Sanchis A, Llopis-González A, MoralesSuárez-Varela M. Evidence of the gluten-free and casein-free diet in autism spectrum disorders: a systematic review. J Child Neurol 2014;29:1718-27.

24. Herndon AC, DiGuiseppi C, Johnson SL, Leiferman J, Reynolds A. Does nutritional intake differ between children with autism spectrum disorders and children with typical development? J Autism Dev Disord 2009;39: 212-22.

25. Sharp WG, Berry RC, McCracken C, et al. Feeding problems and nutrient intake in children with autism spectrum disorders: a meta-analysis and comprehensive review of the literature. J Autism Dev Disord 2013;43. 2159-73.

26. Buie T, Fuchs GJ 3rd, Furuta GT, et al. Recommendations for evaluation and treatment of common gastrointestinal problems in children with ASDs. Pediatrics 2010;125 Suppl 1:S19-29.

27. Anderson RJ, Bendell DJ, Garnett I, et al. Identification of indolyl-3-acryloylglycine in the urine of people with autism. J Pharm Pharmacol 2002;54:295-8.

28. Bull G, Shattock P, Whiteley P, et al. Indolyl-3-acryloylglycine (IAG) is a putative diagnostic urinary marker for autism spectrum disorders. Med Sci Monit 2003;9:CR422-5.

29. Wright B, Brzozowski AM, Calvert E, et al. Is the presence of urinary indolyl-3-acryloylglycine associated with autism spectrum disorder? Dev Med Child Neurol 2005;47:190-2.

30. Wang L, Angley MT, Gerber JP, et al. Is urinary indolyl-3-acryloylglycine a biomarker for autism with gastrointestinal symptoms? Biomarkers 2009;14:596-603.

31. Cook JG. Creatinine assay in the presence of protein. Clin Chim Acta 1971;32:485-6.
32. Mills MJ, Savery D, Shattock PE. Rapid analysis of low levels of indolyl3-acryloylglycine in human urine by high-performance liquid chromatography. J Chromatogr B Biomed Sci Appl 1998;712:51-8.

33. Tilley K, Akhtar M, Gani, D. The stereochemical course of decarboxylation, transamination and elimination reactions catalysed by Escherichia coli glutamic acid decarboxylase. J Chem Soc Perkin Trans 1994;1: 3079-3087.

34. Food and Drug Administration of the United States of America. Guidance for Industry on Bioanalytical Method Validation. Rockville, MD: Center for Drug Evaluation and Research in cooperation with the Center for Veterinary Medicine; Biopharmaceutics Coordinating Committee, 2001.

35. World Health Organization. The ICD-10 classification of mental and behavioural disorders: clinical descriptions and diagnostic guidelines. Geneva: World Health Organization, 1992.

36. Wright B, Williams C, Smith R, et al. An autism spectrum disorders forum: a model for the effective use of multidisciplinary assessment and intervention planning with limited clinical resources. Autism-Open Access 2016;6:1-7.

37. Lord C, Risi S, Lambrecht L, et al. The autism diagnostic observation schedule-generic: a standard measure of social and communication deficits associated with the spectrum of autism. J Autism Dev Disord 2000;30:205-23.

38. Gorrindo P, Williams KC, Lee EB, Walker LS, McGrew SG, Levitt P. Gastrointestinal dysfunction in autism: parental report, clinical evaluation, and associated factors. Autism Res 2012;5:101-8.

39. Molloy CA, Manning-Courtney P. Prevalence of chronic gastrointestinal symptoms in children with autism and autistic spectrum disorders. Autism 2003;7:165-71.

40. Kuschner ES, Eisenberg IW, Orionzi B, et al. A preliminary study of selfreported food selectivity in adolescents and young adults with autism spectrum disorder. Res Autism Spectr Disord 2015;15-16:53-9.

41. Levy SE, Souders MC, Ittenbach RF, Giarelli E, Mulberg AE, Pinto-Martin JA. Relationship of dietary intake to gastrointestinal symptoms in children with autistic spectrum disorders. Biol Psychiatry 2007;61:492-7.

42. Bandini LG, Anderson SE, Curtin C, et al. Food selectivity in children with autism spectrum disorders and typically developing children. J Pediatr 2010;157:259-64.

43. Erickson CA, Stigler KA, Corkins MR, Posey DJ, Fitzgerald JF, McDougle CJ. Gastrointestinal factors in autistic disorder: a critical review. J Autism Dev Disord 2005;35:713-27.

44. Alpert M. The autism diet. Sci Am 2007;296:19-20.

45. Keen DV. Childhood autism, feeding problems and failure to thrive in early infancy. Seven case studies. Eur Child Adolesc Psychiatry 2008;17:209-16. 